\title{
Hepatocyte-Derived Lipocalin 2 Is a Potential Serum Biomarker Reflecting Tumor Burden in Hepatoblastoma
}

\author{
Laura Molina, ${ }^{* \dagger}$ Danielle Bell, ${ }^{\dagger \dagger}$ Junyan Tao, ${ }^{* \dagger}$ Morgan Preziosi, ${ }^{* \dagger}$ Tirthadipa Pradhan-Sundd, ${ }^{* \dagger}$ Sucha Singh, ${ }^{* \dagger}$ \\ Minakshi Poddar, ${ }^{* \dagger}$ Jianhua Luo, ${ }^{* \dagger}$ Sarangarajan Ranganathan, ${ }^{\dagger \S}$ Maria Chikina, ${ }^{\dagger \oplus}$ and Satdarshan P. Monga ${ }^{* \dagger}$
}

\begin{abstract}
From the Division of Experimental Pathology, * Department of Pathology, the Department of Computational and Systems Biology, "and the Division of Gastroenterology, Hepatology and Nutrition," Department of Medicine, University of Pittsburgh School of Medicine, Pittsburgh; the Pittsburgh Liver Research Center, ${ }^{\dagger}$ University of Pittsburgh Medical Center and University of Pittsburgh School of Medicine, Pittsburgh; and the Division of HematologyOncology, ${ }^{\ddagger}$ Department of Pediatrics, and the Division of Pediatric Pathology, ${ }^{\S}$ Department of Pathology, Children's Hospital of Pittsburgh, Pittsburgh, Pennsylvania
\end{abstract}

\author{
Accepted for publication \\ May 3, 2018 \\ Address correspondence to \\ Satdarshan P. Monga, M.D., \\ University of Pittsburgh School \\ of Medicine, 200 Lothrop St., \\ S-422 BST, Pittsburgh, \\ PA 15261. E-mail: smonga@ \\ pitt.edu.
}

\begin{abstract}
Hepatoblastoma (HB) is the most common pediatric liver malignant tumor. Previously, we reported coactivation of $\beta$-catenin and Yes-associated protein-1 (YAP1) in $80 \%$ of HB. Hepatic co-expression of active $\beta$-catenin and YAP1 via sleeping beauty transposon/transposase and hydrodynamic tail vein injection led to HB development in mice. Here, we identify lipocalin 2 (Lcn2) as a target of $\beta$-catenin and YAP1 in HB and show that serum Lcn2 values positively correlated with tumor burden. Lcn2 was strongly expressed in HB tumor cells in our mouse model. A tissue array of 62 HB cases showed highest LCN2 expression in embryonal and lowest in fetal, blastemal, and small cell undifferentiated forms of $\mathrm{HB}$. Knockdown of LCN2 in $\mathrm{HB}$ cells had no effect on cell proliferation but reduced NF- $\kappa \mathrm{B}$ reporter activity. Next, liver-specific Lcn2 knockout (KO) mice were generated. No difference in tumor burden was observed between Lcn2 KO mice and wild-type littermate controls after sleeping beauty transposon/ transposase and hydrodynamic tail vein injection delivery of active YAP1 and $\beta$-catenin, although Lcn2 $\mathrm{KO}$ mice with $\mathrm{HB}$ lacked any serum Lcn2 elevation, demonstrating that transformed hepatocytes are the source of serum Lcn2. More blastemal areas and inflammation were observed within HB in Lcn2 K0 compared with wild-type tumors. In conclusion, Lcn2 expressed in hepatocytes appears to be dispensable for the pathogenesis of HB. However, transformed hepatocytes secrete serum Lcn2, making Lcn2 a valuable biomarker for HB. (Am J Pathol 2018, 188: 1895-1909; https://doi.org/10.1016/ j.ajpath.2018.05.006)
\end{abstract}

Hepatoblastoma (HB) is the most common pediatric liver malignant tumor, comprising about $1 \%$ of all pediatric cancers and accounting for $80 \%$ of pediatric liver cancer. ${ }^{1,2}$ Twenty percent of children will present with lung metastasis at diagnosis, and for this subset of patients, the prognosis is poor. $^{3-5}$ Treatment is dependent on staging, which is derived from a combination of radiographic and histologic findings. Although well-differentiated fetal HB can be cured with resection alone, less differentiated histologic subtypes, such as embryonal or crowded fetal, require more aggressive treatment with cytotoxic chemotherapy followed by surgical resection or liver transplantation. ${ }^{3} \mathrm{HB}$ is unique in that multiple different histologic subtypes can exist within a single tumor. ${ }^{6}$ These histologic differences may be attributable to mutations that occur at various stages of hepatoblast development. ${ }^{7}$ The most well-known mutations in $\mathrm{HB}$ are in CTNNB1 ( $\beta$-catenin gene) and are seen in

\footnotetext{
Supported by NIH grants 1R01DK62277 (S.P.M.), 1R01DK100287 (S.P.M.), and 1R01CA204586 (S.P.M.), Endowed Chair for Experimental Pathology (S.P.M.), and in part by Cellular Approaches to Tissue Engineering and Regeneration grant T32 EB001026 (L.M.).

L.M. and D.B. contributed equally to this work.

Disclosures: None declared.
} 
approximately $90 \%$ of human $\mathrm{HB}^{8-11}$ Recently, overactivation of Yes-associated protein-1 (YAP1) was found in most HB. In fact, co-activation of $\beta$-catenin and YAP1 was reported in $80 \%$ of human $\mathrm{HB}^{12}$ It is presumed that $\beta$ catenin and YAP1 activation results in nuclear translocation and binding to transcription factors T-cell factor 4 (TCF4) and TEA domain (TEAD), respectively, to initiate cell proliferation, growth, and differentiation. ${ }^{13-15}$

Previously, we developed a murine model of HB using sleeping beauty transposon/transposase and hydrodynamic tail vein injection (SB-HTVI) to overexpress constitutively active forms of $\beta$-catenin $(\triangle \mathrm{N} 90-\beta$-catenin) and YAP1 (YAPS127A) in hepatocytes. ${ }^{16}$ Co-expression of these two proto-oncogenes but not single expression led to $\mathrm{HB}$ development in YAP1- $\beta$-catenin mouse model with $100 \%$ penetrance. ${ }^{12,16}$ This finding suggests that an oncogenic promoter downstream of $\beta$-catenin and YAP1 may be involved in HB development. Here, we identify Lcn2 as one gene regulated by both $\beta$-catenin and YAP1 and up-regulated in $\mathrm{HB}$ in mice and patients.

Lipocalin 2 (Lcn2), alias neutrophil gelatinase-associated lipocalin (NGAL), is a small protein that has been implicated in a variety of human cancers, including breast, pancreatic, gastric, colon, ovarian, and hepatocellular carcinomas. ${ }^{17-20} \mathrm{It}$ was first discovered less than a decade ago as a component of the innate immune system, present in neutrophils and other inflammatory cells as well as the epithelial lining of the respiratory and gastrointestinal tracts. Len2 participates in iron metabolism by binding siderophores and moving iron intracellularly and can deprive bacteria of iron, causing decreased bacterial growth. ${ }^{21}$ It is also a secretory protein present in both serum and urine of humans and animals and has been studied as a potential biomarker for acute kidney injury. ${ }^{22-24}$ The role of Lcn2 in cancer has recently been under close scrutiny. Lcn2 is overexpressed in breast cancer and even more so in metastatic disease, directly correlating with worse patient outcomes. ${ }^{25-27}$ Conversely, overexpression of Lcn2 in pancreatic cancer is associated with antitumorigenic effect. $^{28,29}$ The mechanisms by which Lcn2 may be involved in oncogenesis have yet to be determined. Lcn2 can bind to matrix metallopeptidase- 9 and stabilize it to aid in tumor invasion and metastasis. ${ }^{18,26}$ Lcn2 induction may trigger inflammation within the tumor environment via the NF- $\mathrm{BB}$ pathway. ${ }^{30-32}$ Lcn 2 may also regulate iron-dependent pathways that are essential for cancer growth. ${ }^{33}$ Increasingly, Lcn 2 has been identified as a nonspecific biomarker of inflammation and organ injury that could be useful in disease monitoring or even predictive of prognosis. ${ }^{20}$

Although Lcn2 has been found to be overexpressed in hepatocellular carcinoma, it has not been studied in HB and not known to be associated with $\beta$-catenin or YAP1 signaling. On the basis of our analysis depicting marked up-regulation of $L c n 2$ in the mouse model of HB, we hypothesized that Lcn2 would be overexpressed in murine and human $\mathrm{HB}$ samples. We also examined whether $\operatorname{Lcn} 2$ was contributing to $\beta$-catenin-YAP1-induced HB. Lcn2 expression in human
HB tumors correlated with tumor differentiation, with increased expression in less differentiated tumors. Lcn2 was secreted from hepatocytes in murine $\mathrm{HB}$, and serum Lcn2 correlated strongly with tumor burden. Although knocking out $L c n 2$ from hepatocytes in mice did not affect HB initiation or progression, it affected overall tumor inflammation and led to areas of distinct histologic findings. Our work offers strong support for the applicability of serum Lcn2 as a biomarker for HB burden and histologic subtypes.

\section{Materials and Methods}

\section{Microarray, Genome-Wide Scan, and Commonly Up- Regulated Genes}

Macroscopic tumor nodules $<7$ weeks after YAP1- $\beta$-catenin injection ( $n=3$ per mouse; three mice) and normal livers from noninjected mice $(n=3)$ were used for mRNA isolation and subjected to Affymetrix gene array using chromatin immunoprecipitation (ChIP) R430 version 2.0 (Affymetrix, Santa Clara, CA). The full data set is available at Gene Expression Omnibus (http://www.ncbi.nlm.nih.gov/geo; accession number GSE112485). Microarray data were normalized using the expresso function from the affy Bioconductor package. ${ }^{34}$ Probes were mapped to genes using the custom brain array CDF. ${ }^{35}$ Differential expression was determined using the limma Bioconductor package, with robust linear models. ${ }^{36} \mathrm{~A}$ list of genes that were significantly (q value $<0.1$ ) up-regulated (at least 1.5 -fold) in tumor samples relative to control livers was compiled. Genome-wide scan for TCF4-TEAD target genes was performed to identify genes containing both TEAD and TCF4 binding sites in their promoters. Potential TCF4 targets were taken from a combination of genome-wide TCF4 ChIP experiments as compiled by the ChIP Enrichment Analysis (CHEA) database. ${ }^{37}$ Because the CHEA database does not contain any data for TEAD, potential TEAD targets were extracted from a genome-wide scan of TEAD consensus sites as provided by the Homer Known Motifs genome track. ${ }^{38}$ Genes that had a TEAD consensus site within 2000 upstream and 500 downstream of the transcription start site were considered. Using the above two approaches, a significant overlap of genes was found (hypergeometric $P=0.01$ ), resulting in five top candidates, of which $L c n 2$ was one and was chosen for additional, in-depth analysis.

\section{In Vivo Studies}

All mouse studies were performed in accordance with NIH's Guide for the Care and Use of Laboratory Animals ${ }^{39}$ and under an approved animal protocol by the Institutional Animal Use and Care Committee at the University of Pittsburgh. Lcn2-floxed mice were used with permission from Dr. Jack Cowland and generously supplied by Dr. Grace Gao (Rutgers University, New Brunswick, NJ). Lcn2- 
floxed mice were crossed with albumin-Cre mice to generate liver-specific Lcn2 knockout (KO) or Lcn2 KO mice. Lcn2 KO mice or wildtype (WT) littermate controls were injected with plasmids for both $\Delta \mathrm{N} 90-\beta$-catenin and YAP S127A at 7 to 8 weeks of age using the concentrations and HTVI technique described in our original publication. ${ }^{16}$ At 6 to 7 weeks post injection, the mice were sacrificed. Serum and livers were harvested from all animals and stored at $-80^{\circ} \mathrm{C}$. Liver sections were placed in $10 \%$ formalin for 48 hours and then transferred to $70 \%$ ethanol. Sections were processed and embedded into paraffin blocks.

\section{Protein Extraction and Western Blot}

After transfection, the cell media was aspirated, and radioimmunoprecipitation assay buffer that contained Protease Inhibitor Cocktail (Thermo Fisher Scientific, Waltham, MA) was added to each well. HepG2 cells were lyzed in radioimmunoprecipitation assay buffer, and three wells of each sample were pooled for optimal protein concentration, which was determined by bicinchoninic acid assay (Thermo Fisher Scientific). Aliquots of $40 \mu \mathrm{g}$ were denatured by boiling for 9 minutes in $2 \times$ Laemmli Sample Buffer (BioRad, Hercules, CA) and loaded in precast $4 \%$ to $15 \%$ gradient gels (Bio-Rad) for electrophoresis. Protein was transferred onto a nitrocellulose membrane via a semidry transfer method using the Trans-Blot Turbo transfer system (Bio-Rad). Membranes were blocked in 5\% nonfat dry milk in blotto (Tris-buffered saline that contained $0.1 \%$ Tween 20) overnight at $4^{\circ} \mathrm{C}$. The primary antibodies used were as follows: rabbit monoclonal anti-LCN2 (ab125075, 1:1000, Abcam, Cambridge, MA), rabbit monoclonal anti-Yap (ab52771, 1:1000, Abcam), mouse monoclonal anti- $\beta$ catenin (1:1000, BD Biosciences, San Jose, CA), rabbit polyclonal anti-p65 (1:200, Santa Cruz Biotechnology, Dallas, TX), rabbit polyclonal phosphor-Ser536-p65 (1:1000, Cell Signaling, Danvers, MA), and mouse monoclonal anti-glyceraldehyde-3-phosphate dehydrogenase (GAPDH) (1:1000, Sigma-Aldrich, St. Louis, MO). Each primary antibody was incubated overnight at $4^{\circ} \mathrm{C}$, except anti-GAPDH, which was added for 2 hours at room temperature. Membranes were washed with blotto and incubated with rabbit $(1: 15,000)$ or mouse $(1: 50,000)$ horseradish peroxidase-secondary antibody for 2 hours. Bands were visualized by Super Signal West Femto kit (Thermo Fisher Scientific).

\section{RNA Extraction and Real-Time PCR}

Frozen livers from a previously published $\mathrm{YAP}-\beta$-catenin model were used. ${ }^{16}$ RNA was extracted with Trizol (Ambion, Carlsbad, CA). A DNase kit (Ambion) was used to remove contaminating genomic DNA and cDNA synthesis kit (Invitrogen, Carlsbad, CA) was used for reverse transcription. Real-time PCR was performed on cDNA using SybrGreen. Primers were purchased from Integrated DNA Technologies
(Coralville, IA). Mouse-specific Lcn2 primers used were as follows: $5^{\prime}$-TTTCACCCGCTTTGCCAACT-3' (forward), 5'-GTCTCTGCGCATCCCAFTCA-3' (reverse). Additional primers used include the following: inducible nitric oxide synthase: 5'-TCACGCTTGGGTCTTGTTCA-3' (forward) and $5^{\prime}$-GGGGAGCCATTTTGGTGACT-3' (reverse); MYC: $5^{\prime}$-CCTAGTGCTGCATGAGGAGA-3' (forward) and $5^{\prime}$ TCCACAGACACCACATCAATTT-3' (reverse); IL-6: 5' ATCAGGAAATTTGCCTATTGAAA-3' (forward) and $5^{\prime}$ CCAGGTAGCTATGGTACTCCAGA-3' (reverse); interferon- $\gamma$ : 5'-ATCTGGAGGAACTGGCAAAA-3' (forward) and $5^{\prime}$-TTCAAGACTTCAAAGAGTCTGAGGTA-3' (reverse); and IL-1 $\beta$ : 5'-AGTTGACGGACCCCAAAAG-3' (forward) and $5^{\prime}$-AGCTGGATGCTCTCATCAGG-3' (reverse). Mousespecific GAPDH primers used were as follows: $5^{\prime}$ AACTTTGGCATTGTGGAAGG-3' (forward) and 5'-ACACATTGGGGGTAGGAACA-3' (reverse).

\section{Serum ELISA Analysis}

Blood from all mice was collected at time of sacrifice. Serum was then used for enzyme-linked immunosorbent assay (ELISA) for Lcn2 using the Mouse Lipocal-2/NGAL Quantikine ELISA kit (catalog number MLCN20; R\&D Systems, Minneapolis, MN). Assays were performed as per manufacturer's instructions.

\section{Immunohistochemistry}

All patient sample analysis was performed under an exempt protocol by the Institutional Review Board at the Children's Hospital of Pittsburgh. A tissue array of 69 deidentified HB cases collected from the Children's Hospital of Pittsburgh was assessed for LCN2 expression using immunohistochemistry. Slides were deparaffinized by passing through xylene, graded alcohol, and deionized water. ${ }^{16}$ Slides were rinsed with phosphate-buffered saline, immersed in citrate buffer at a $\mathrm{pH}$ of 6.0 , and microwaved at $60 \%$ power. Three percent hydrogen peroxide was applied for 10 minutes, and slides were rinsed with phosphate-buffered saline. Samples were blocked for 30 minutes using Superblock (ScyTek Laboratories Inc., Logan, UT) and incubated with LCN2 antibody (HPA002695, 1:100, Sigma-Aldrich) at room temperature for 60 minutes. Slides were rinsed and incubated with biotin-conjugated secondary rabbit antibody (Chemicon, Temecula, CA) for 30 minutes. Signal was detected using Vectastain ABC Elite kit (Vector Laboratories, Inc., Burlingame, CA) and developed with diaminobenzidine (Vector Laboratories). All slides were then counterstained with Shandon hematoxylin solution (Thermo Fisher Scientific). Slides were dehydrated using graded alcohol $(95 \%, 100 \%)$ and xylene before being coverslipped.

The tissue array was reviewed by a pediatric pathologist (S.R.) and signal positivity determined using a 0 - to 3-point grading scale, with zero indicating no expression and 3 being highest expression. Samples of normal liver included 
on the same tissue array were used as baseline controls. The histologic subgroup of each sample was determined by cell morphologic features and arrangement. LCN2 cytoplasmic and nuclear immunoreactivity was recorded for each histologic component of each sample.

The same immunohistochemistry protocol was used to analyze the expression of several markers in paraffinembedded mouse liver samples. The following antibodies were used: Myc-tag (CS2276S, 1:1000, Cell Signaling, Danvers, MA), proliferating cell nuclear antigen (sc-56, 1:4000, Santa Cruz Biotechnology, Santa Cruz, CA), cyclinD1 (ab134175, 1:200, Abcam), glutamine synthetase (GS; G2781, 1:4000, Sigma-Aldrich), CD45 (sc-53665, 1:100, Santa Cruz Biotechnology), F4/80 (MCA497GA, 1:100, Serotech, Hercules, CA), and neutrophil elastase (ab68672, 1:1500, Abcam).

\section{Cell Culture, Transfection, and in Vitro Assays}

HepG2 cells, a human HB cell line with a known 116 -amino acid deletion in $\beta$-catenin, were grown in Eagle's minimum essential media, supplemented with $10 \%$ fetal bovine serum. Fifty percent confluent HepG2 cells plated in 6-well plates $(200,000$ cells per well) were transfected with siRNA against LCN2 (catalog number AM51331; identification number 121013; Ambion), $\beta$-catenin (catalog number 6225s; Cell Signaling Technology), Yap1 (catalog number 4392420; identification number s20368; Ambion), or scrambled RNA (catalog number AM4611; Ambion) using INTERFERin siRNA Transfection Reagent (catalog number 409-10; Polyplus Transfection, Illkirch, France) as recommended by the manufacturer and incubated for 72 hours. All knock-downs were performed in triplicate.

A tritium-labeled thymidine incorporation assay was performed on transfected HepG2 cells. Two hundred thousand cells per well in six-well plates in triplicate were transfected with siRNA against $L C N 2$ or scrambled control. Twenty-four hours later, $1 \mathrm{~mL}$ per well of Eagle's minimum essential media that contained $2.5 \mu \mathrm{Ci}$ of ${ }^{3} \mathrm{H}$-thymidine (PerkinElmer, Waltham, MA) was added to the plate. After 24 or 48 hours, cells were washed with cold 5\% trichloroacetic acid, washed with cold running tap water, and dried. One milliliter of 0.33 $\mathrm{mol} / \mathrm{L} \mathrm{NaOH}$ was added next for 30 minutes. Three hundred microliters of cell solution was added to scintillation vial, mixed with $3 \mathrm{~mL}$ of Universal Scintillation Fluid (PerkinElmer), and placed in dark for 30 minutes; Then the radioactivity measured with a scintillation counter.

To assess p65 transcriptional regulatory activity, HepG2 cells were seeded in sixwell plates and transiently transfected with plasmids or siRNA combined with reporters. Validated LCN2 siRNA or negative control siRNA (Ambion) was used at a final concentration of $25 \mathrm{nmol} / \mathrm{L}$ in the presence of Lipofectamine 3000 reagent (Invitrogen), as per the manufacturer's instructions. Simultaneously, $0.4 \mu \mathrm{g}$ of p65 luciferase reporter plasmid (provided by Dr. Kari
Nejak-Bowen, University of Pittsburgh, Pittsburgh, PA) was co-transfected with $0.1 \mu \mathrm{g}$ of Renilla plasmid, also in the presence of Lipofectamine $3000 .^{40}$ Cells were harvested 48 hours after transfection for protein extraction and luciferase assay. Luciferase assay was performed using the Dual Luciferase Assay System kit (Promega, Madison, WI). Relative luciferase activity (in arbitrary units) was reported as fold-induction after normalization for transfection efficiency. All studies were performed in triplicate.

\section{Results}

Genome-Wide Scan for TCF4-TEAD Target Genes and Mouse HB Analysis Reveals LCN2 as a Candidate Target of YAP1 and $\beta$-Catenin in This Tumor Type

In a previous study, SB-HTVI was used to co-express constitutively active $\beta$-catenin $(\Delta \mathrm{N} 90 \beta$-catenin) and YAP1 (YAPS127A) in mouse liver to generate the YAP1- $\beta$-catenin model, which resulted in rapid development of HB in these mice. ${ }^{16}$ Because both proto-oncogenes regulate target gene expression via binding to their respective transcription factors, candidate targets commonly regulated by the two pathways, which may have a role in HB pathogenesis, were explored. In silico analysis was performed to identify such genes. Potential TCF4 targets were taken from a combination of genome-wide TCF4 ChIP experiments as compiled by the CHEA database. ${ }^{37}$ Three thousand forty-five genes were identified as TCF4 targets (Figure 1A). Because the CHEA database does not contain any data for TEAD, potential TEAD targets were extracted from a genome-wide scan of TEAD consensus sites; 2422 genes were identified as potential TEAD targets (Figure 1A). ${ }^{38}$

Next, tumor-bearing livers from the YAP1- $\beta$-catenin and age-matched WT FVB livers were used for mRNA isolation and Affymetrix gene array using ChIP R430 version 2.0 as described in Materials and Methods. Fifty-nine genes were found to be up-regulated $>1.5$-fold in expression in YAP1- $\beta$ catenin livers compared with the controls (Figure 1A).

Next, the information obtained from the above approaches was combined to identify overlap between genes having $\beta$-catenin-TCF and YAP1-TEAD binding in their promoter regions and were also up-regulated in the YAP1- $\beta$-catenin model. A significant overlap of genes (hypergeometric $P=0.01$ ) was found, resulting in five top candidates (Figure 1A). One of these genes was Lcn2.

To verify that Lcn2 was indeed overexpressed in the YAP1- $\beta$-catenin model, three additional tumor-bearing livers and three WT livers were used for mRNA isolation for realtime PCR analysis. A significant up-regulation (approximately 600-fold) in Lcn2 expression was observed in HBbearing livers in the YAP1- $\beta$-catenin mice (Figure 1B).

Because Lcn 2 is a secreted protein, its levels were tested in the serum of control and YAP1- $\beta$-catenin mice. Control FVB mice had approximately $100 \mathrm{ng} / \mathrm{mL}$ of $\mathrm{Lcn} 2$ in serum (Figure 1C). Serum from YAP1- $\beta$-catenin mice collected at 
A

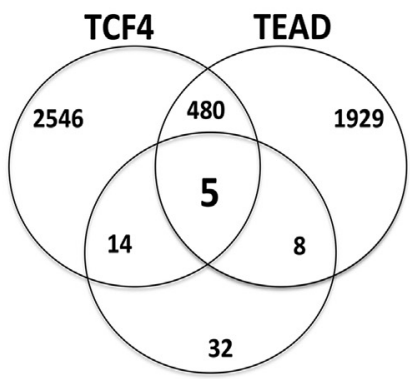

HB up-regulated
B

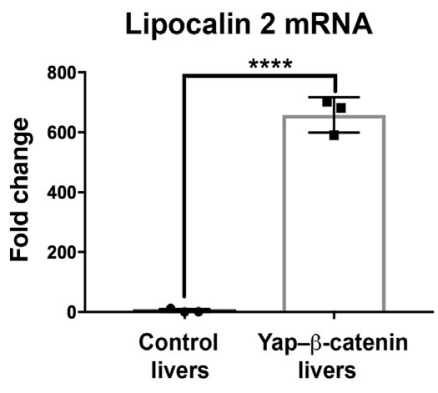

C

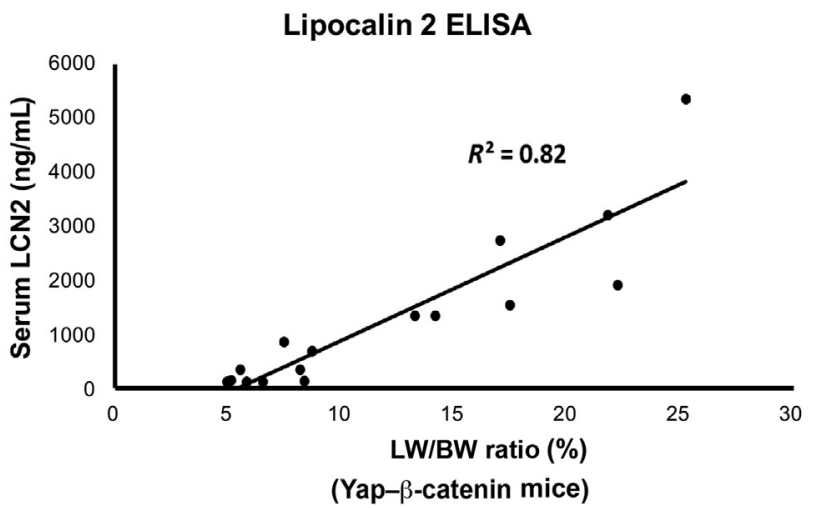

D

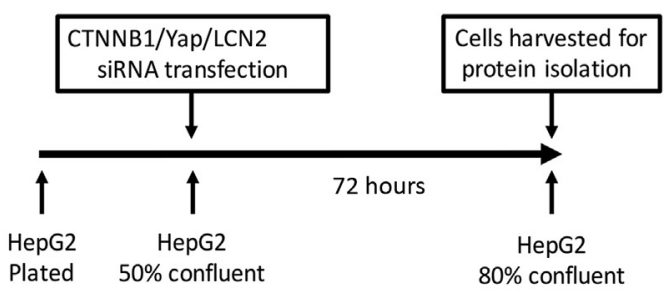

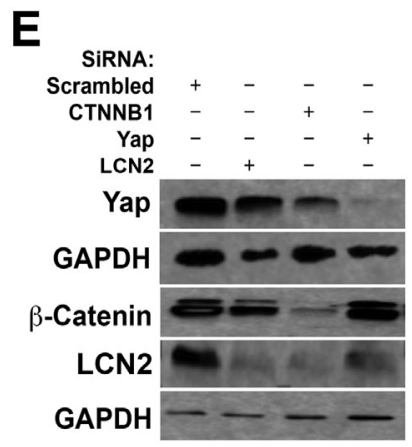

Figure 1 Lipocalin 2 (LCN2) is regulated by Yes-associated protein-1 (YAP1)- $\beta$-catenin in hepatoblastomas (HBs). A: Venn diagram of up-regulated genes in a mouse genome analysis illustrating that five gene loci that contain binding sites for T-cell factor 4 (TCF4) and TEA domain (TEAD) also have upregulated expression in murine HB. B: Realtime PCR of liver lysates from control mouse livers and $\beta$-catenin-YAP1-induced HB tumor-laden livers, showing significantly increased expression of LCN2. C: Serum enzyme-linked immunosorbent assay (ELISA) for LCN2 was performed on mice with HB tumors and varying degrees of tumor burden, approximated by liver weight-to-body weight (LW/BW) ratio, showing a positive and significant correlation between serum LCN2 level and disease burden in mice $\left(R^{2}=0.82\right)$. D: Schematic representation of the timeline for HepG2 cell transfection with silencing RNA targeting $\beta$-catenin, YAP1, LCN2, or scrambled SiRNA. E: Western blot shows decreased levels of LCN2 after transfection of HepG2 cells with SiRNA to LCN2, $\beta$-catenin, or YAP1. Efficacy of each of the siRNA is shown by decrease in their respective protein levels. Glyceraldehyde3-phosphate dehydrogenase (GAPDH) was used as a loading control. ${ }^{* * *} P<0.0001$ (t-test). time of sacrifice during a 5- to 10 -week postinjection period had a dramatic increase with progressive tumorigenesis, with some animals having approximately $5000 \mathrm{ng} / \mathrm{mL}$ of Lcn2 (Figure 1C). Because YAP1- $\beta$-catenin mice had significantly increased liver weight-to-body weight (LW/BW) ratios representing increased tumor burden, it was next tested whether the serum Lcn2 levels in these mice correlated with tumor burden. Indeed, serum Lcn2 values were directly proportional to the LW/BW ratio of the animals with a coefficient of determination $\left(R^{2}\right)$ of 0.82 (Figure 1C). Thus, these results demonstrate a strong correlation between serum Lcn2 levels and tumor burden in this mouse model of HB.

\section{YAP1 and $\beta$-Catenin Regulate Expression of LCN2 in HB} Cells

To further confirm that LCN2 expression is regulated by both $\beta$-catenin and YAP1, changes in LCN2 protein levels were analyzed after siRNA-mediated knockdown of $\beta$-catenin or
YAP1 in the human HepG2 HB cell line (Figure 1D). Western blot analysis using HepG2 cells transfected with control, YAP1, or $\beta$-catenin siRNA showed that knockdown of $\beta$-catenin or YAP1 independently caused a notable decrease in total LCN2 levels (Figure 1E). Interestingly, a more pronounced effect of $\beta$-catenin suppression than YAP1 suppression was evident on LCN2 levels. To address whether LCN2 silencing in HepG2 cells would have any effect on YAP1 or $\beta$-catenin levels, these cells were also transfected with siRNA against $L C N 2$ (Figure 1E). Although $L C N 2$ suppression was evident, its knockdown did not affect the levels of $\beta$-catenin or YAP1 in the HepG2 cells (Figure 1E). Thus, $\beta$-catenin and YAP1 regulate $L C N 2$ expression in the HB cells.

\section{LCN2 Is Aberrantly Expressed in HB in Patients and Correlates with Tumor Histologic Subtype}

Because approximately $80 \%$ of HBs have concomitant nuclear $\beta$-catenin and YAP $1{ }^{16}$ and LCN2 is regulated by both of 
these oncoproteins, immunohistochemistry was performed for LCN2 on a tissue array of HB samples collected from Children's Hospital of Pittsburgh. Of the 69 patient samples that were stained for LCN2, seven were excluded because of the loss of tissue during the staining process, leaving 62 patient samples for analysis. Each sample was classified as having one or more of the eight different histologic components by an experienced pediatric pathologist (S.R.) based on the classification parameters described in proceedings from the Children's Oncology Group International Pathology Symposium in $2014 .{ }^{41}$ In fact, within the tissue array, $69 \%$ of tumors exhibited more than one histologic subtype, and some tumor samples had up to five subtypes. Deidentified patient demographic information and the full list of histologic classifications found in each patient's tumor is given in Supplemental Table S1. To account for differences in LCN2 positivity based on histologic classification, the expression of LCN2 was quantified in all histologic subtypes independently identified within each tumor. Although the total number of patients was 69 , the total number of distinct histologic samples identified for analysis was 160 , broken down into 43 embryonal (27\%), 53 crowded fetal (33\%), 20 fetal (12.5\%), 18 blastema (11\%), 11 small cell undifferentiated (7\%), 8 epithelial-mesenchymal transitional (EMT) (5\%), 4 teratoid (2.5\%), and 3 giant cell (2\%) (Supplemental Figure S1A). Each sample was then graded based on the nuclear and cytoplasmic expression of LCN2 as observed by immunohistochemistry, ranging from absent (0), mild (1), moderate (2), to strong (3). Within the tissue array, several samples of normal pediatric liver adjacent to tumor sections were used as a negative control (grade 0 ) because LCN2 is not expressed in epithelium in the normal liver tissue (Supplemental Figure S1B). Within each sample, Kupffer cells, which are inflammatory cells that innately express LCN2, were used as a positive control (grade 3). In fact, in normal human liver, Kupffer cells expressed LCN2 at baseline by immunohistochemistry (Supplemental Figure S1B).

On analysis of HB cases, all embryonal components of HB across various cases had nuclear or cytoplasmic staining with typical intensity of 2 to 3 (Figures 2 and 3, A and B). Ninety-five percent of the crowded fetal tumor samples also had positive staining for LCN2, but with a lower intensity of 1 to 2 . In contrast, only $50 \%$ of the most differentiated, or fetal, HBs were positive for LCN2, with absent nuclear LCN2 and a typical cytoplasmic stain intensity of 1 to 2 (Figures 2 and 3, A and B). Notably, all blastemal tumor samples, which have a stromal cell origin, ${ }^{41}$ were negative for LCN2. Similarly, small cell undifferentiated tumors, which have neither epithelial nor mesenchymal differentiation, ${ }^{41}$ had the second lowest expression levels of LCN2, with only $18 \%$ of samples staining positive for LCN2 (Figures 2 and 3, A and B). Interestingly, teratoid tumors, which are rare tumors that often contain heterologous cellular elements from multiple germ layers, had $100 \%$ positive staining for LCN2 (Figures 2 and 3, A and B).

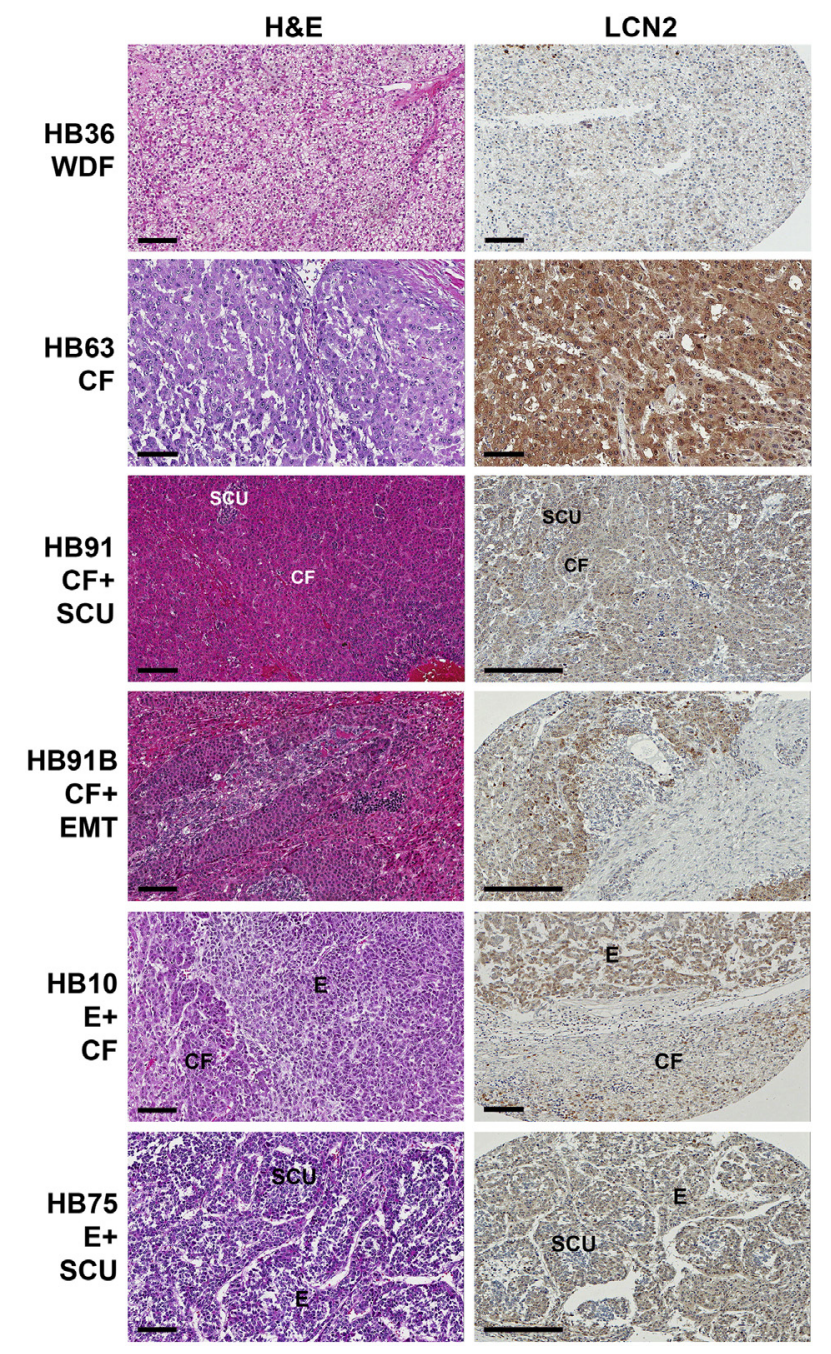

Figure 2 Immunohistochemical analysis of lipocalin 2 (LCN2) expression in hepatoblastoma (HB) cases. Representative images from human HB samples with hematoxylin and eosin (H\&E) staining and LCN2 immunohistochemistry. Note that the intensity of staining is dependent on histologic subtype, which is exemplified in HB75, where small cell undifferentiated (SCU) islands of hepatoblasts have no LCN2 expression compared with the surrounding embryonal hepatoblasts, which are positive for LCN2. LCN2 is negative in the well-differentiated fetal histologic subtype of HB36 and the epithelial-mesenchymal (EMT) subtype of HB91B. Scale bars: $100 \mu \mathrm{m}$ (short bars); $200 \mu \mathrm{m}$ (long bars). CF, crowded fetal; $\mathrm{E}$, embryonal; WDF, well-differentiated fetal.

These results demonstrate that LCN2 expression differs greatly based on the histologic subtype of $\mathrm{HB}$ and correlates with the level of tumor cell differentiation. Figure 3A shows the proportion of tumors of each histologic subtype that exhibited positive staining for LCN2. Figure 3B further breaks down the data to show the distribution of scores of LCN2 stain intensity, revealing cytoplasmic expression in the outer circle and nuclear expression in the inner circle. Taken together, these results reveal a dramatic correlation between the expression of LCN2 and the tumor grade of $\mathrm{HB}$, with the highest LCN2 expression in embryonal and crowded fetal tumor samples as well as teratoid and giant cell tumors. 
A

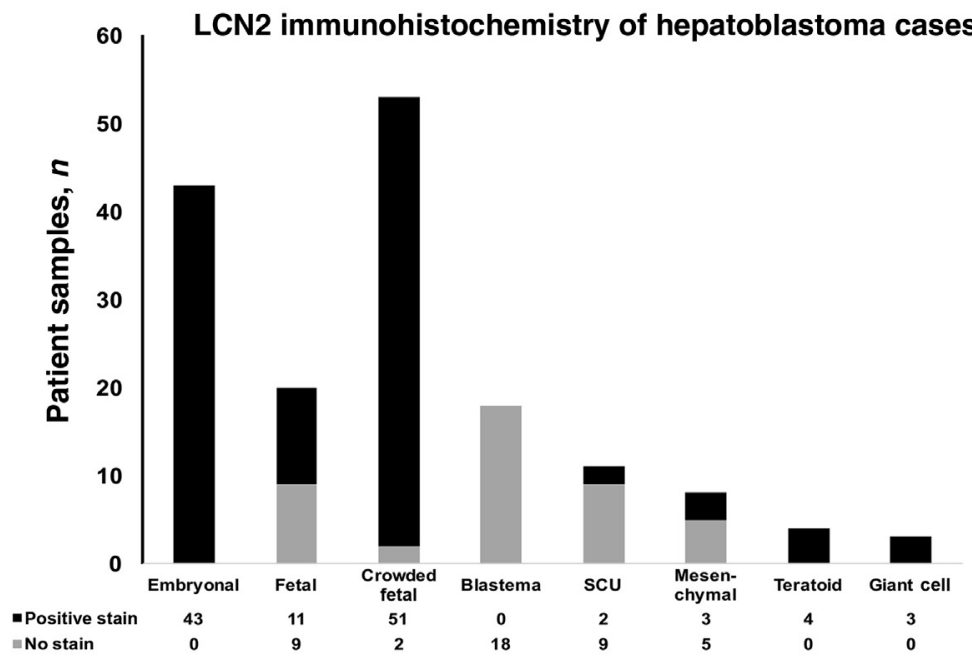

B

Fetal staining $(n=20)$

$\square$ Absent $=$ Mild $=$ Moderate $=$ Strong

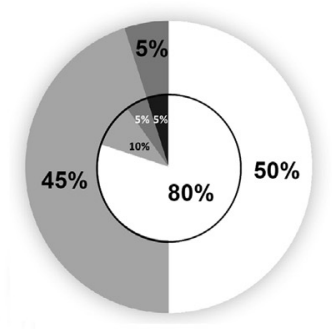

Crowded fetal staining ( $n=53$ )

$\square$ Absent $=$ Mild $=$ Moderate - Strong

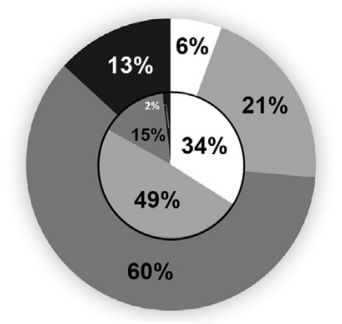

Embryonic staining ( $n=53$ ) $\square$ Absent $"$ Mild $=$ Moderate - Strong

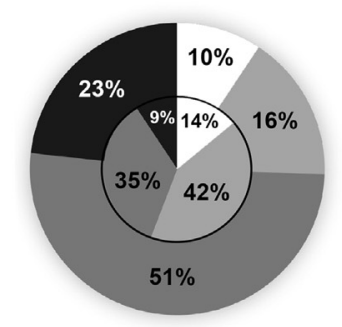

C

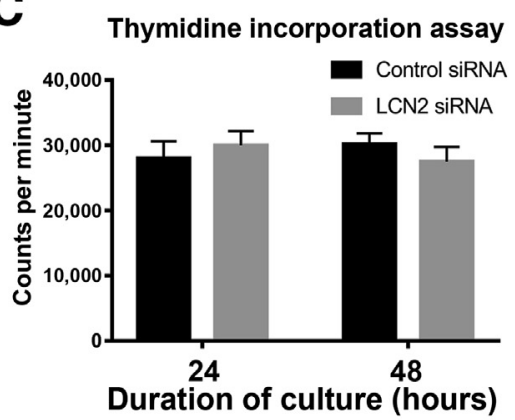

D

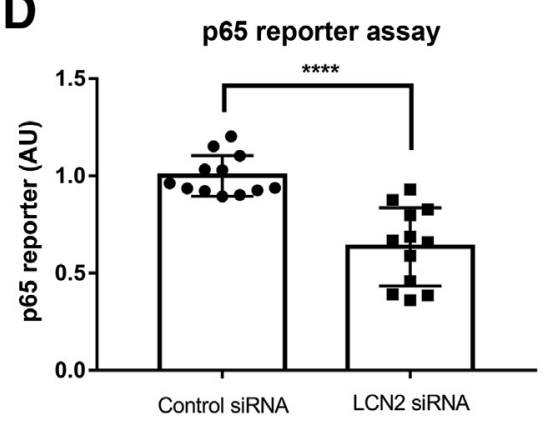

Figure 3 Expression of lipocalin 2 (LCN2) in hepatoblastoma (HB) cases and its role in HB cells. A: Tumor samples with positive staining (including grades 1 to 3 ) for each different histologic subtype found in the collection of HB samples from Children's Hospital of Pittsburgh. B: Distribution of nuclear LCN2 staining (inner circle) and cytoplasmic LCN2 staining (outer circle) among the three most common subtypes of $\mathrm{HB}$, including absent (0), mild (1), moderate (2), and strong (3) staining intensity. Embryonal, which is the least differentiated, has the most nuclear staining and the highest intensity of LCN2 expression. Fetal histologic subtype is well differentiated and has only $50 \%$ cytoplasmic staining, very little nuclear staining, and less intense LCN2 expression. Crowded fetal histologic subtype is less differentiated than fetal tumors but more so than embryonal tumors and has intermediate staining intensity. C: Thymidine incorporation assay was performed 24 to 48 hours after treatment of HepG2 cells with scrambled siRNA or siRNA targeting $L C N 2$, showing no difference between the two groups. D: Results of a luciferase reporter assay to detect the transcriptional regulatory activity of p65 show a significant decrease in luciferase activity in HepG2 cells after 48-hour treatment with LCN2 siRNA compared with scrambled siRNA ${ }^{*} P<0.0001$ ( $t$-test). SCU, small cell undifferentiated.

\section{Effect of LCN2 Silencing on HepG2 Cell Proliferation and p65 Reporter Activity}

Because LCN2 was strongly expressed in tumor cells within $\mathrm{HB}$, its biological role was investigated next. LCN2 was silenced in a HB cell line, and its effect on cell proliferation was queried using thymidine incorporation. Interestingly, at 24 or 48 hours, no difference in thymidine incorporation was observed in HepG2 cells transfected with either control of LCN2-specific siRNA (Figure 3C). This finding suggests that LCN2 does not play a direct role in regulating $\mathrm{HB}$ cell proliferation.
The effect of LCN2 was next studied on gene expression regulation in HepG2 cells because LCN2 was present in the nucleus of tumor cells in patients. Studies have found that LCN2 is involved in a regulatory feedback loop with NF- $\kappa \mathrm{B}$ to control the level of inflammation in chronic injury. ${ }^{42} \mathrm{~A}$ p65 luciferase reporter plasmid was used to assess the activity of $\mathrm{NF}-\kappa \mathrm{B}$. Treatment of HepG2 cells with siRNA targeting LCN2 mRNA led to a significant decrease in p65 luciferase reporter activity, suggesting that LCN2 may be inducing NF- $\kappa \mathrm{B}$ activity in $\mathrm{HB}$ cells (Figure 3D). Thus, LCN2 does not directly regulate cell proliferation. However, it positively regulates NF- $\kappa \mathrm{B}$ signaling in HB cells. 
Liver-Specific Lcn2 Conditional KO Mice Lack Any Overt Phenotype

To more conclusively address the role of Len2 in YAP1- $\beta$ catenin-driven $\mathrm{HB}$ and examine its potential role as a secreted biomarker that represents tumor burden in this model, a liver-specific Lcn $2 \mathrm{KO}$ model was generated using Lcn2-floxed and albumin-Cre transgenic mice. These mice were verified as Lcn2 $\mathrm{KO}$ by the presence of the homozygous Lcn2-floxed alleles and Cre-recombinase in PCR analysis of genomic DNA (Figure 4A). For all studies, littermates with homozygous Lcn2-floxed alleles and absent Cre were used as controls and henceforth referred to as WT mice. The Lcn2 KO mice were born in normal mendelian ratio and lacked any overt phenotype. Real-time PCR using RNA isolated from WT and Lcn2 KO livers showed a significant decrease in Lcn2 expression in the Lcn2 KO mice, although variable expression was observed in WT mice (Figure 4B). The LW/BW ratio was comparable between the two groups (Figure 4C). Serum analysis found no abnormalities in alanine aminotransferase, total bilirubin, or alkaline phosphatase (Figure 4, D-F). The results of hematoxylin and eosin staining of liver sections were unremarkable for Len $2 \mathrm{KO}$ and WT mice (Figure $4 \mathrm{G}$ ).

\section{Co-Expression of Constitutively Active YAP1 and} $\beta$-Catenin in Lcn2 K0 Mice via SB-HTVI Does Not Alter the Growth and Development of HB

To address whether Lcn 2 is playing a role in $\mathrm{HB}$ development in the YAP1- $\beta$-catenin model, YAP1 and $\beta$-catenin were coexpressed via SB-HTVI in the Lcn $2 \mathrm{KO}$ and WT mice. By 6 to
A

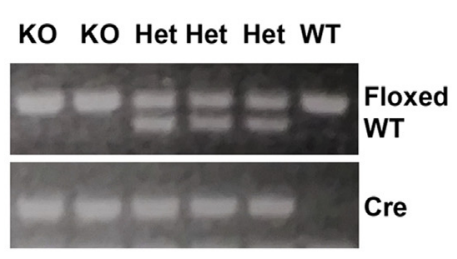

C

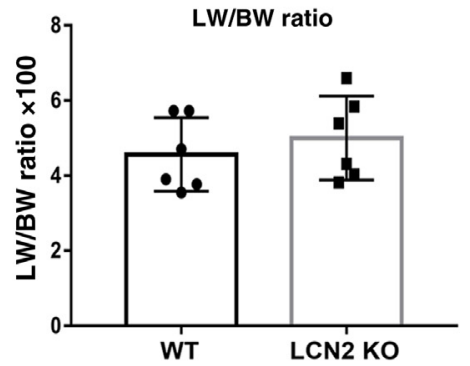

E

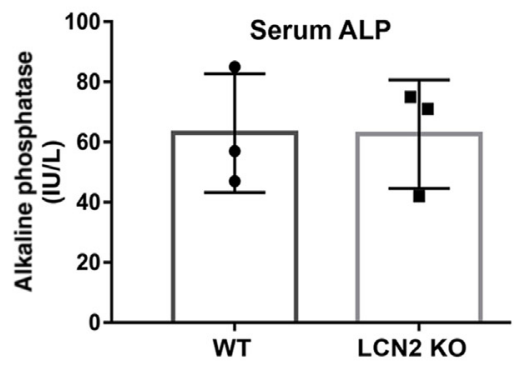

G

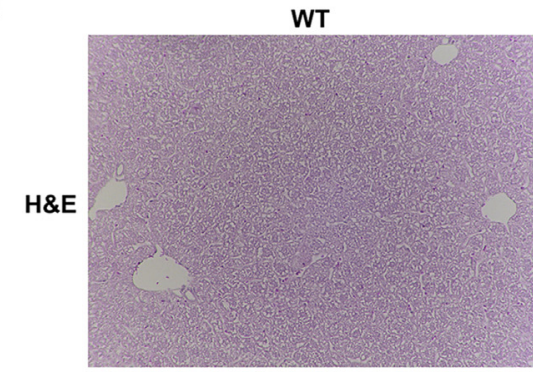

B

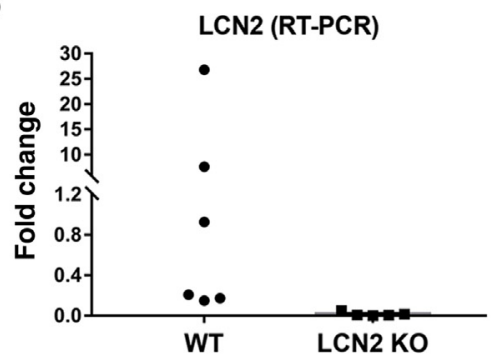

D

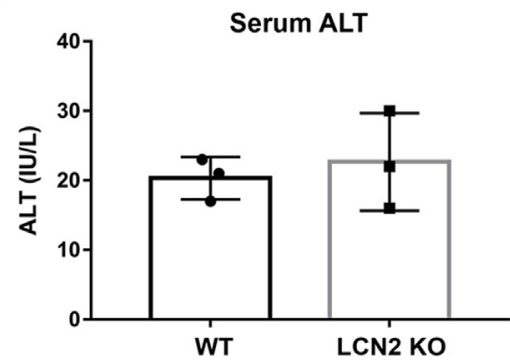

$\mathbf{F}$

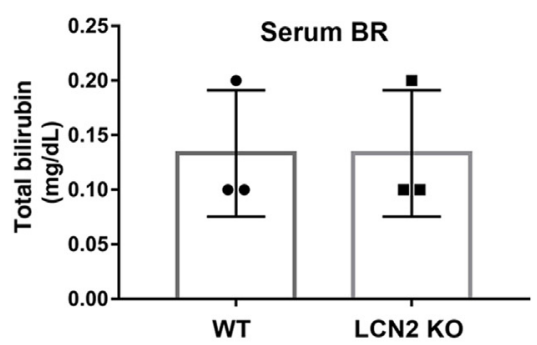

LCN2 KO

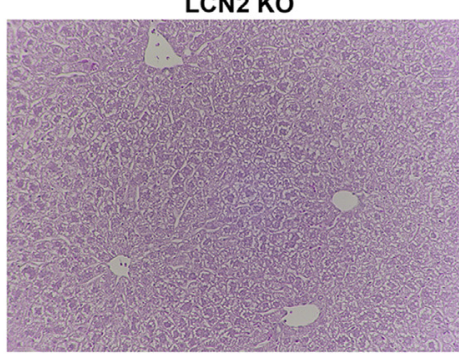

Figure 4 Generation and characterization of liver-specific Lcn2 knockout mice. A: Genotyping of mouse littermates distinguishes between wildtype (WT) mice lacking Cre expression, mice heterozygous for the Lcn2-floxed allele (Het), and lipocalin 2 (Lcn2) knockout (KO) mice homozygous for the Lcn2-floxed allele and positive for Cre expression. B: Quantitative RT-PCR for Lcn2 confirms decreased Ln2 expression in Lcn2 KO mouse livers compared with WT livers. C: Liver weight-to -body weight (LW/BW) ratios of control and liverspecific Lcn2 KO mice at baseline. Liver function tests, including serum alanine aminotransferase (ALT) (D), alkaline phosphatase (ALP) (E), and total serum bilirubin (BR) $(\mathbf{F})$, revealed no difference between WT and Lcn2 KO mice. G: Representative hematoxylin and eosin (H\&E) stains of WT and Lcn2 KO mice. Original magnification, $\times 100(\mathbf{G})$. AU, arbitrary units. 
7 weeks post injection, Lcn2 $\mathrm{KO}$ and WT mice had large and grossly comparable tumors (Figure 5A). This finding was also reflected by the lack of any significant difference in the LW/ $\mathrm{BW}$ ratio between the $\mathrm{Lcn} 2 \mathrm{KO}$ and WT mice administered YAP1- $\beta$-catenin through SB-HTVI (Figure 5B). Hematoxylin and eosin staining of representative sections show tumors occurring in Lcn2 $\mathrm{KO}$ mice and controls after YAP1- $\beta$ catenin, which were reminiscent of crowded fetal or fetal HB as in the original YAP1- $\beta$-catenin model (Figure 5C). Although no major differences were evident, there was evidence of more crowded fetal histologic components and more pronounced areas of blastemal cells within tumors in the Lcn2 KO mice compared with the WT mice (Figure 5C). To confirm that tumors in both groups were composed of cells that expressed exogenous YAP1 and $\beta$-catenin, the sections were stained for Myc-tag, which represents exogenous
A

$$
\text { Yap1- } \beta \text {-catenin }
$$

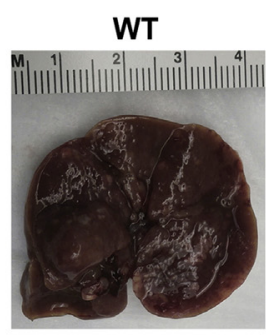

C

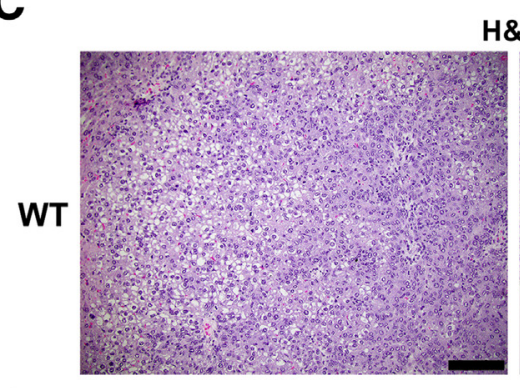

H\&E

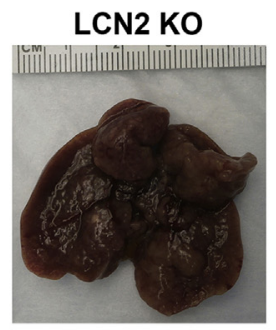

B
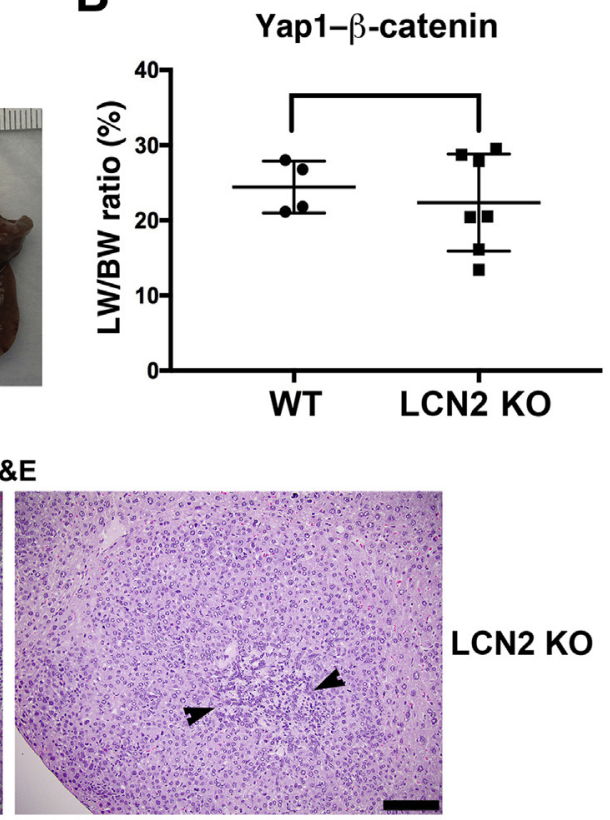

D

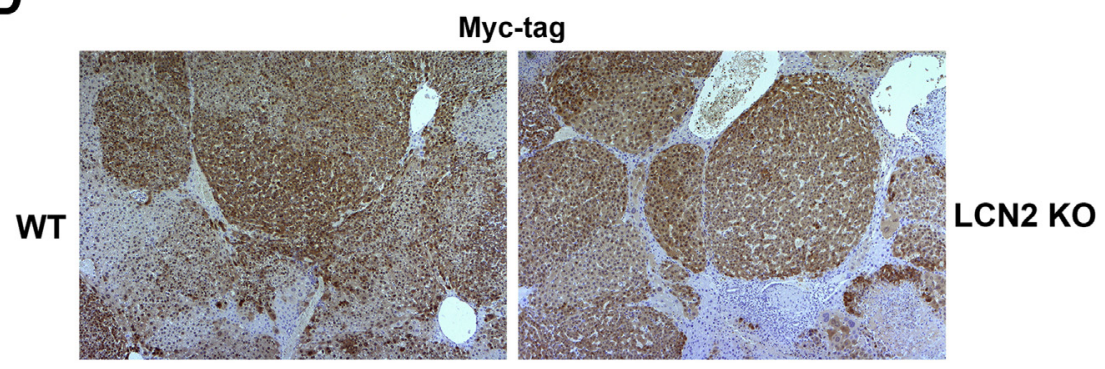

E

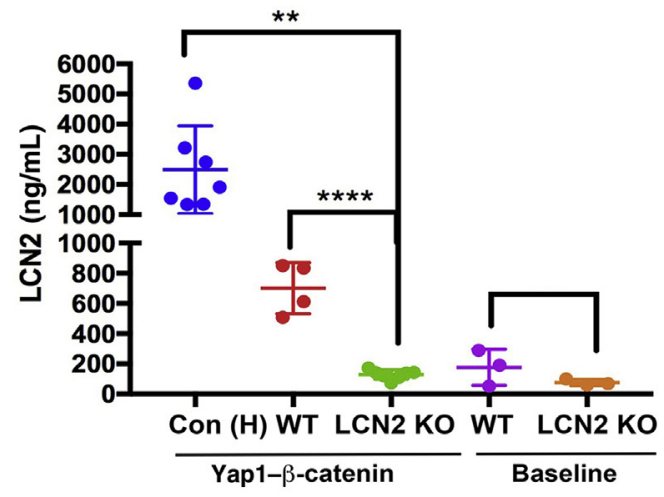

Figure 5 Hepatoblastoma (HB) development in lipocalin 2 (LCn2) knockout (KO) versus wildtype (WT) mice after Yes-associated protein-1 (YAP1)- $\beta$-catenin expression. A: Representative gross images of WT and Lcn2 K0 livers 6 to 7 weeks after HB induction. B: Liver weight-to-body weight (LW/BW) ratios of control and Lcn2 KO mice at 6 to 7 weeks after induction of HB. C: Representative hematoxylin and eosin (H\&E) stains of murine $\mathrm{HB}$ in control and Lcn2 $\mathrm{KO}$ mice showing relatively more blastemal component (arrowheads). D: Immunohistochemistry for MycTag shows strongly positive tumor nodules in WT and Lcn2 K0 mice treated with YAP1- $\beta$-catenin by sleeping beauty transposon/transposase and hydrodynamic tail vein injection (SB-HTVI). E: Serum Lcn2 levels detected by enzyme-linked immunosorbent assay of control mice at baseline (C57BL) $6 \mathrm{~J}$ background; purple), Lcn2 $\mathrm{KO}$ mice at baseline (orange), littermate WT mice 6 to 7 weeks after HB induction [LW/BW ratios $<10 \%$ (red)], and historical controls [Con $(\mathrm{H})]$ after 8 to 10 weeks of SB-HTVI injection and LW/BW ratios $>10 \%$ (blue). ${ }^{* *} P<0.01,{ }^{* * * * P}<0.0001$. Scale bars $=100$ $\mu \mathrm{m}$. Original magnification, $\times 100$ (D). 


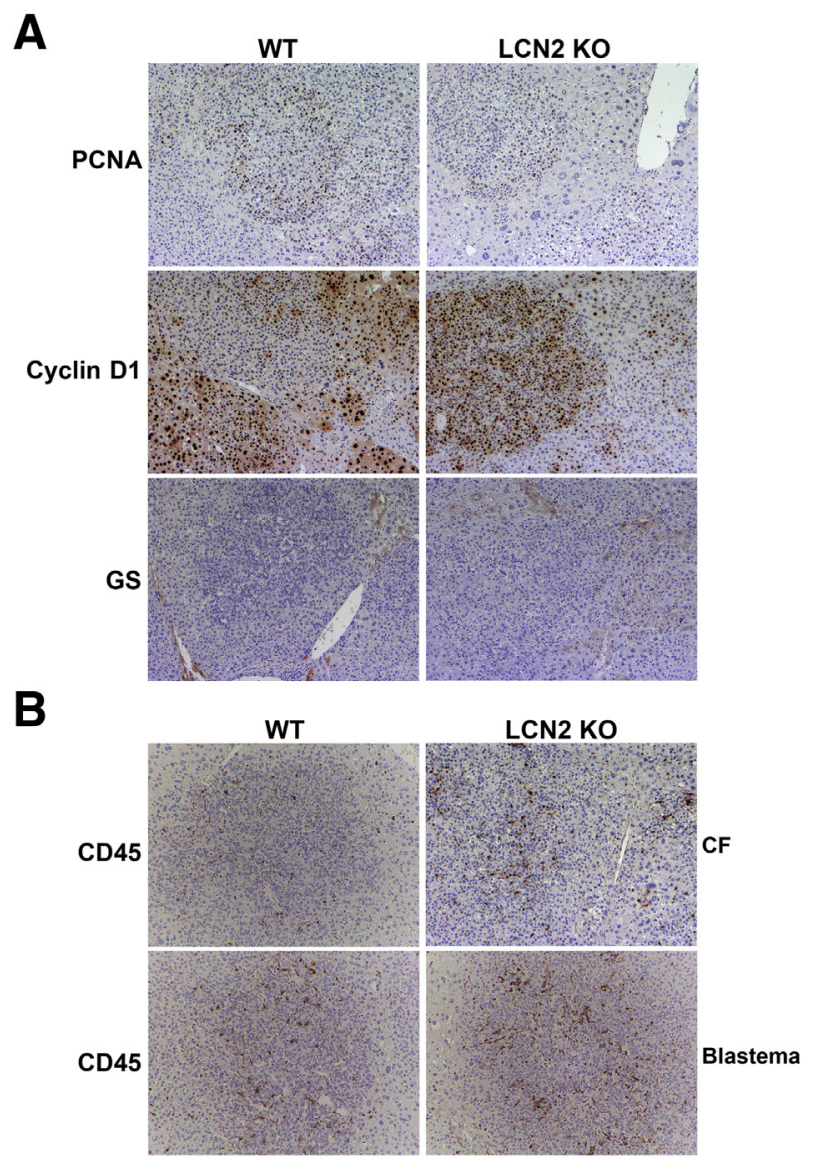

Figure 6 Differences in hepatoblastoma (HB) occurring in lipocalin 2 (Lcn2) knockout (KO) versus wild-type (WT) mice after Yes-associated protein-1 (YAP1)- $\beta$-catenin expression. A: Representative immunohistochemistry for proliferating cell nuclear antigen (PCNA), cyclin D1, and glutamine synthetase (CS) in WT and Lcn2 KO livers 6 to 7 weeks after HB induction. B: Representative immunohistochemistry for CD45 in WT and Lcn2 K0 livers 6 to 7 weeks after HB induction showing modest increase in overall inflammation in Lcn2 $\mathrm{KO}$ mice. Blastemal areas in WT and Lcn2 KO HB shows an increase in CD45-positive cells in these areas, but numbers are more pronounced in Lcn2 KO mice. Original magnification, $\times 100(\mathbf{A}$ and $\mathbf{B})$. CF, crowded fetal.

$\beta$-catenin SB plasmid. As expected, the tumors were strongly positive for Myc-tag in WT and KO mice (Figure 5D).

Serum Lcn2 Is Derived from Hepatocytes in YAP1- $\beta$ Catenin HB Murine Model

Because serum Lcn 2 correlated strongly with tumor burden in the YAP1- $\beta$-catenin HB model but tumor burden was comparable between YAP1 $-\beta$-catenin injected Lcn $2 \mathrm{KO}$ and WT mice, it could be conclusively tested whether the elevated serum Lcn 2 in this model was indeed being secreted by the transformed hepatocytes, especially because inflammatory cells are the known source of Lcn2. ELISA for Lcn2 was performed on serum from baseline Lcn2 KO mice, littermate WT controls, YAP1 $-\beta$-catenin injected tumor harboring Ln2 $\mathrm{KO}$ mice, and WT as well as YAP1- $\beta$-catenin historical controls with advanced HB. Serum Lcn2 levels were very low in WT and Len2 KO mice at baseline with insignificant differences (Figure 5E). The low levels of serum Lcn2 $(<200 \mathrm{ng} /$ $\mathrm{mL}$ ) at baseline and absence of differences in serum Lcn2 in WT versus Lcn $2 \mathrm{KO}$ mice indicates that hepatocytes are not the source of basal serum Lcn2. A marked and significant reduction in serum Lcn 2 level was observed in $\mathrm{Lcn} 2 \mathrm{KO}$ mice at 6 to 7 weeks (mean, $150 \mathrm{ng} / \mathrm{mL}$ ) after YAP1- $\beta$-catenin compared with the time-matched, YAP1- $\beta$-catenin-injected WT mice, which had approximately $700 \mathrm{ng} / \mathrm{mL}$ of Lcn2 (Figure 4E). The highest levels of Lcn2 (mean, $2500 \mathrm{ng} / \mathrm{mL}$ ) were observed in historical controls, which represent advanced stages of HB development and the greatest tumor burden as

A

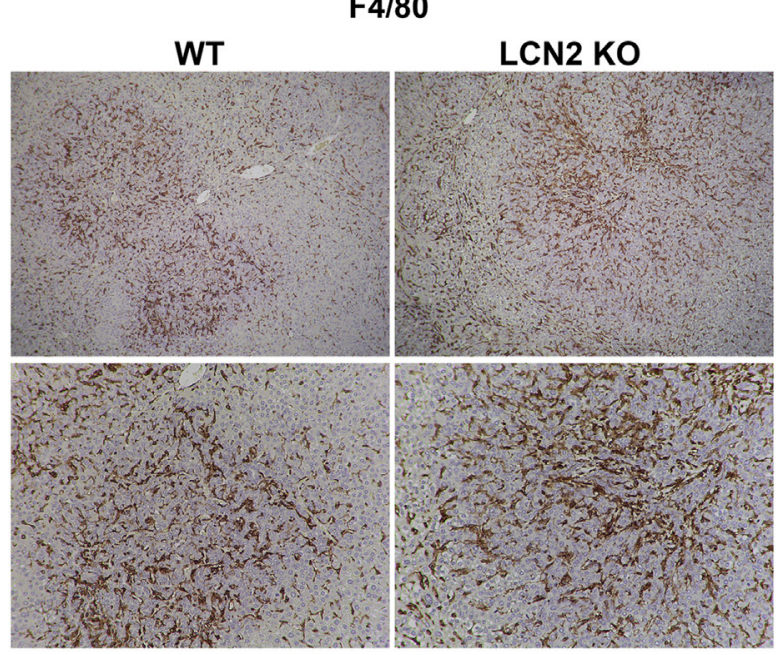

B

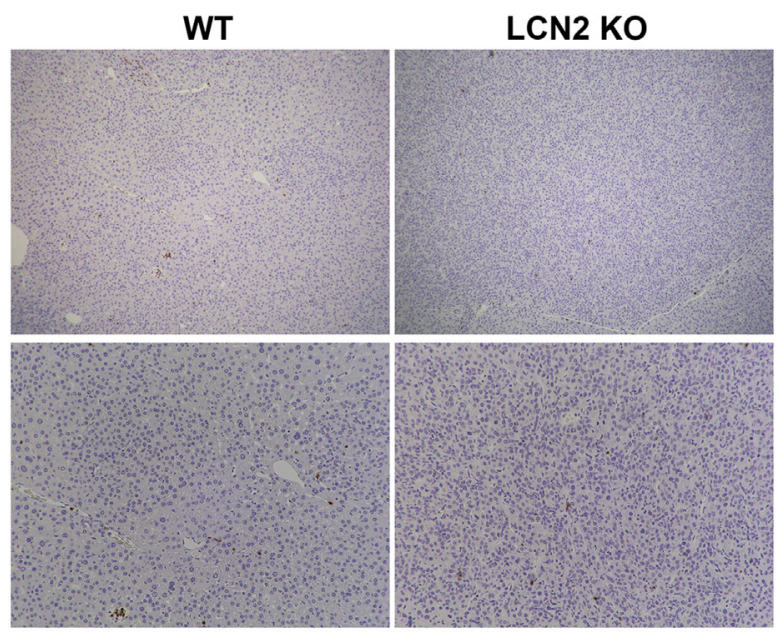

Figure 7 Differences in macrophage and neutrophil infiltration in hepatoblastoma (HB) occurring in lipocalin 2 (LCn2) knockout (KO) versus wild-type (WT) mice after Yes-associated protein-1 (YAP1)- $\beta$-catenin expression. A: Representative immunohistochemistry for the macrophage marker $\mathrm{F} 4 / 80$ showing large numbers of macrophages infiltrating $\mathrm{HB}$ tumors in both Yap1- $\beta$-catenin-driven tumors in WT and Len2 KO livers. B: Representative immunohistochemistry for the neutrophil marker neutrophil elastase showing a few neutrophils infiltrating the Yap1- $\beta$ catenin-driven HB tumors in WT and LCn2 KO livers. Original magnification: $\times 50$ (A and $\mathbf{B}$, top rows); $\times 100$ (A and $\mathbf{B}$, bottom rows). 
A

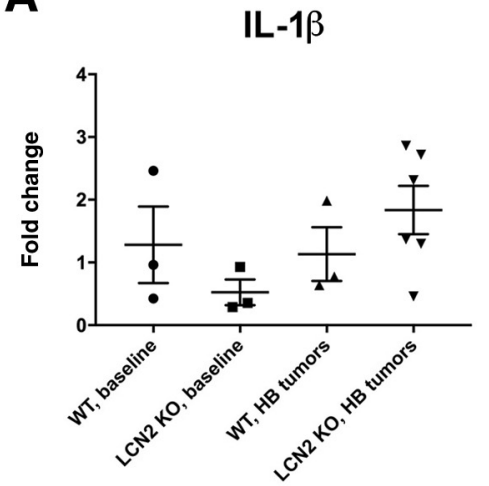

IL-6

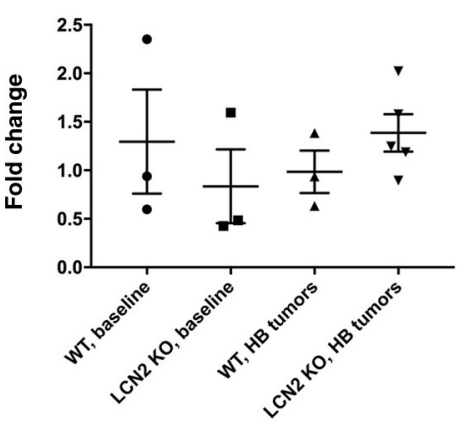

IFN- $\gamma$

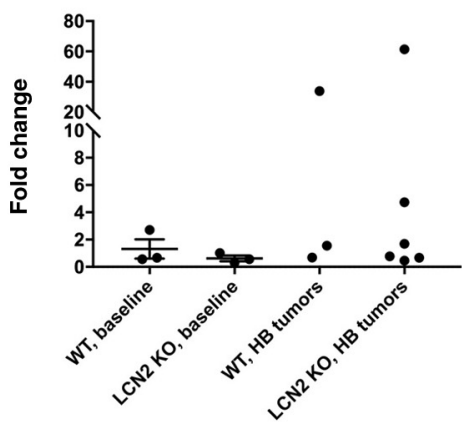

B
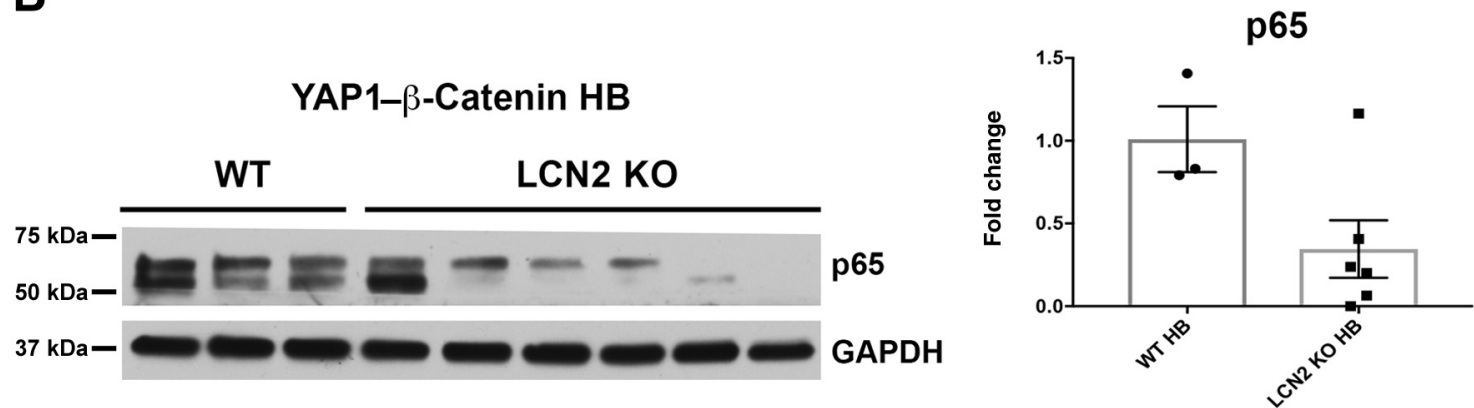

YAP1- $\beta$-Catenin HB
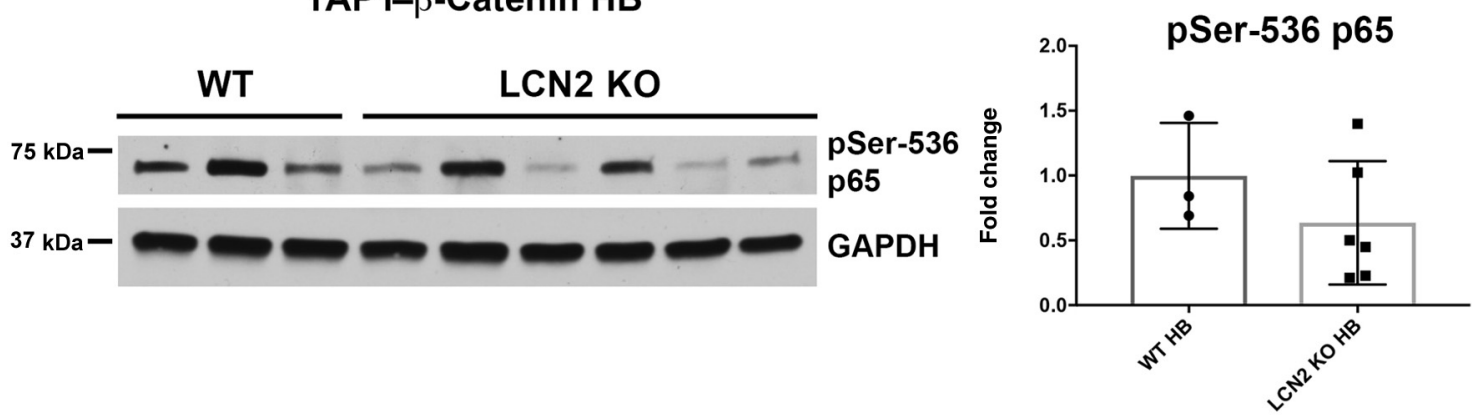

C

FAS

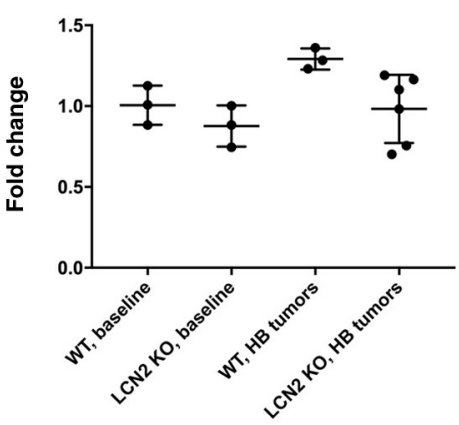

iNOS

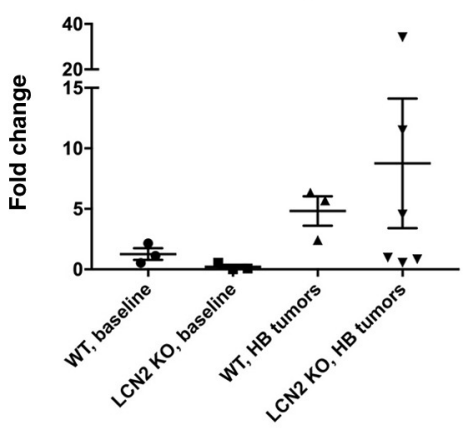

Myc

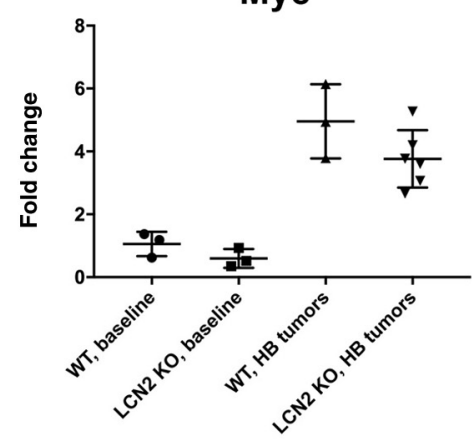

Figure 8 Evaluating inflammatory cytokines and NF- $\kappa$ B signaling in hepatoblastoma (HB) occurring in lipocalin 2 (Lcn2) knockout (K0) versus wild-type (WT) mice after Yes-associated protein-1 (YAP1)- $\beta$-catenin co-expression. A: Gene expression of inflammatory cytokines IL-1 $\beta$, IL-6, and interferon (IFN)- $\gamma$ was detected using real time-PCR, showing high levels of variability within tumor-laden livers of both WT and Lcn2 K0 HBs. No significant difference in cytokine expression is detected in baseline WT and Lcn2 KO livers. B: Lcn2 K0 HB shows decreased total levels of p65 and the activated phospho-Ser536 p65 by Western blot. Protein levels were quantified using densitometry. There is no statistically significant difference between WT and Lcn2 KO HBs. C: Gene expression of p65 targets FAS, inducible nitric oxide synthase (iNOS), and Myc was detected using real-time PCR, showing high levels of variability within tumor-laden livers of both WT and Lcn2 KO HBs. No significant difference in gene expression is detected in baseline WT and Lcn2 KO livers. GAPDH, glyceraldehyde-3-phosphate dehydrogenase. 
reflected by a $>10 \% \mathrm{LW} / \mathrm{BW}$ ratio (Figure $5 \mathrm{E}$ ). The marked reduction in serum $L \mathrm{cn} 2$ in the Lcn $2 \mathrm{KO}$ mice after YAP1- $\beta$ catenin injection clearly establishes that tumor cells in the HB are the source of serum Lcn2, supporting its potential as a biomarker for HB disease burden.

Differences in Histologic Components, Inflammation, and p65 Activity in YAP1- $\beta$-Catenin-Induced HB in Lcn2 KO versus WT Mice

To further address the role of Lcn2 in vivo in HB pathogenesis, YAP1- $\beta$-catenin-induced $\mathrm{HB}$ in Lcn2 $\mathrm{KO}$ and WT mice was assessed for cell proliferation. No difference between the two groups was observed as shown by immunohistochemistry for proliferating cell nuclear antigen (Figure 6A). Likewise, tumors in both groups were strongly positive for cyclin-D1 (Figure 6A). The expression of the $\beta$-catenin target GS was next assessed. YAP1- $\beta$-catenininduced HB is GS negative. ${ }^{16} \mathrm{HB}$ occurring in both Lcn2 $\mathrm{KO}$ and WT mice continued to be GS negative (Figure 6A).

On the basis of the in vitro evidence showing that knocking down Len2 decreased p65 transcriptional activity, the role of $\mathrm{Lcn} 2$ in modulating inflammatory response during HB development was studied. The presence of inflammation was evaluated by performing immunohistochemistry for CD45, a pan-leukocyte marker. Lcn2 KO tumors exhibited a modest increase in the overall numbers of CD45-positive cells compared with WT tumors (Figure 6B). Both the WT and Lcn2 KO tumors showed increased presence of CD45-positive cells in areas of blastemal histologic features (Figure 6B), which were more profound and frequent in Lcn2 $\mathrm{KO}$ mice.

Immunohistochemistry was performed for the macrophage marker F4/80 and the neutrophil marker neutrophil elastase to determine the distribution of innate immune cell types in the tumor microenvironment. Macrophages were abundant in the tumor microenvironment in WT and Lcn2 $\mathrm{KO}$ tumors (Figure 7A). A comparison of the abundance and distribution of F4/80 and CD45 staining suggests that macrophages comprise most immune cells in the HB tumor model. Few neutrophils were detected in both WT and Lcn2 KO tumors, except in areas of necrosis within large tumors (Figure 7B). No notable differences were evident in the overall numbers of macrophages or neutrophils between the WT and Lcn2 KO tumors despite marginal increase in overall CD45-positive cells in Lcn2 KO tumors. Likewise, variable expression of various inflammatory markers, including IL-6, IL-1 $\beta$, and interferon- $\gamma$, was also observed in the two groups, both at baseline and in the tumor-bearing livers from Yap- $\beta$-catenin WT and Lcn2 KO mice (Figure 8A).

Activity of $\mathrm{p} 65$ in vivo in the WT and Lcn2 KO tumors was investigated using whole liver samples. Interestingly, the protein levels of p65 trended downward in the Lcn2 KO tumors, as did the levels of the activated phospho-Ser536-p65, although the quantified results are not statistically significant
(Figure 8B). The expression of various cytokines that are known targets of NF- $\mathrm{BB}$ signaling was also determined. However, a high variability in the expression of various such targets, including Fas, inducible nitric oxide synthase, and Myc, was observed by quantitative RT-PCR analysis using mRNA from tumor-bearing livers from Yap- $\beta$-catenin WT and Lcn2 KO mice (Figure 8C). No differences were observed in the expression of NF- $\kappa \mathrm{B}$ targets at baseline between the WT and Lcn2 KO mice (Figure 8C).

Thus, overall, SB-HTVI injection of mutated YAP1- $\beta$ catenin led to the induction of similar HB tumors with mostly crowded fetal histologic subtype in WT and Alb-Cre Lcn2 KO mice, but Lcn2 KO tumors had a modest increase in intratumoral inflammation and more frequent areas of blastemal histologic components.

\section{Discussion}

The current study offers novel insight into the role of LCN2 in $\mathrm{HB}$. We found that $L c n 2$ expression is driven by YAP1 and $\beta$-catenin, the primary tumor drivers in mouse model of $\mathrm{HB}$ and most HB cases. Serum levels of Lcn2 correlated strongly with tumor burden, and it is evident that serum Lcn2 is derived from tumor cells within murine HB. Notably, LCN2 is present in most human $\mathrm{HB}$, and levels of LCN2 expression show an intriguing correlation with the histologic subtypes within individual tumors. Together, this evidence presents LCN2 as a clinically relevant biomarker of $\mathrm{HB}$ tumor burden.

LCN2 is already under investigation in clinical trials as a biomarker for a variety of solid tumors, and assays for serum and urine LCN2 are readily available for use in clinical laboratories. Recent studies have found that serum and urine LCN2 concentrations may have diagnostic and prognostic utility in colorectal, pancreatic, and thyroid cancer. ${ }^{20,43,44}$ Future studies using patient serum samples will be important to assess LCN2 expression and whether LCN2 can be used in conjunction with $\alpha$-fetoprotein to aid in the diagnosis, surveillance, prognosis, and even therapeutic response of HB. Serum and urine LCN2 levels are also elevated in a variety of liver diseases, mirroring the progression of inflammatory and oxidative damage to the liver. ${ }^{45}$ Clinical trials using LCN2 as a serum biomarker for acute kidney injury have also found that serum LCN2 levels are strongly influenced by underlying inflammatory processes, preexisting kidney injury, and other comorbidities in severely ill patients, which may confound its use as a biomarker in patients receiving toxic chemotherapy. ${ }^{46}$ Nevertheless, the same studies have found an increased predictive capacity in neonates and pediatric patients, who are more likely to have few comorbidities and normal renal function. Thus, serum LCN2 holds promise as a clinical tool to improve detection and monitoring of $\mathrm{HB}$ in children.

Most human tissue samples used in this study were taken from patients who had already received some form of chemotherapy and who were referred to Children's Hospital 
of Pittsburgh with late-stage disease because of its status as a major tertiary care and transplant center. The results showing a strong correlation of LCN2 nuclear and cytoplasmic staining with tumor differentiation status are thus most applicable to late-stage HB tumors. It is unclear how chemotherapy may affect LCN2 expression in HB tumors, and further studies are needed to expand these results to a larger group of pretreatment HB tumors to account for this confounding variable. A larger, prospective patient cohort would also be needed to query whether LCN2 expression in early-stage disease may be correlated to measures of prognosis and survival in children with HB.

Despite these limitations, this study sheds some light on the role of LCN2 in HB pathobiology. Within the patient cohort, all embryonal cases and most crowded fetal cases of HB had the highest level of expression of LCN2. However, loss of Lcn2 in hepatocytes did not affect HB proliferation in vitro or in vivo, suggesting that Lcn2 may not directly influence cell proliferation and that the more undifferentiated cases of HB may just have the highest activity of YAP1 and $\beta$-catenin. ${ }^{47}$ Although there are conflicting reports in the literature regarding the potential role of Lcn2 in regulating hepatocyte proliferation after liver injury, our results are consistent with studies that found no difference in proliferation between WT and Lcn2 KO hepatocytes during liver regeneration. ${ }^{48,49}$ YAP1 and $\beta$-catenin promote hepatocyte proliferation through a variety of downstream targets independent of Lcn2, so it is not surprising that loss of Lcn2 alone in hepatocytes may not alter the level of proliferation in tumor cells. Increased frequency of blastemal (stromal) areas was also observed in the HB derived from Lcn2deficient hepatocytes, which may be attributable to a yet unknown role of Lcn 2 in regulating stem cell differentiation, which requires additional studies. In addition, EMT may contribute to blastemal-like phenotype. However, conflicting evidence indicates that Lcn2 inhibits EMT in hepatocellular carcinoma and colorectal cancer while promoting tumor invasion, metastasis, and EMT in prostate and breast cancer. ${ }^{19,29,50-52}$ Again, additional studies will be necessary to address any role of Lcn2 in EMT in HB.

LCN2 silencing in HB cells in vitro affected NF- $\kappa \mathrm{B}$ transcriptional activity, which is consistent with a prior study. ${ }^{42}$ However, the results in vivo were ambiguous, although a downward trend in total and active p65 was noted. The heterogeneity in amounts of tumors in lysates from tumorbearing livers and the presence of Lcn2-expressing inflammatory cells in the tumor microenvironment in WT and Lcn2-KO may be contributing to the high level of variability in p65 levels and activity in whole liver lysates in protein and mRNA expression analysis. It is likely though that $\mathrm{Lcn} 2$ in $\mathrm{HB}$ may be regulating NF- $\kappa \mathrm{B}$ to in turn regulate inflammation in HB. Indeed, we observed a marginal increase in overall infiltrating immune cells in Lcn2 $\mathrm{KO} \mathrm{HB}$, especially in blastemal areas, despite comparably higher macrophage numbers and low neutrophils in both experimental groups. A complex association between p65 and $\beta$-catenin, upstream effector of Lcn 2 has also been noted. ${ }^{40}$ Future studies will address how Lcn2 may be influencing specific immune response and the overall histologic features of HB.

\section{Acknowledgments}

Lcn2-floxed mice were generously supplied by Dr. Grace Gao (Rutgers University, New Brunswick, NJ) and p65 luciferase reporter plasmid was provided by Dr. Kari NejakBowen (University of Pittsburgh, Pittsburgh, PA).

\section{Supplemental Data}

Supplemental material for this article can be found at https://doi.org/10.1016/j.ajpath.2018.05.006.

\section{References}

1. McLaughlin CC, Baptiste MS, Schymura MJ, Nasca PC, Zdeb MS: Maternal and infant birth characteristics and hepatoblastoma. Am J Epidemiol 2006, 163:818-828

2. Darbari A, Sabin KM, Shapiro CN, Schwarz KB: Epidemiology of primary hepatic malignancies in U.S. children. Hepatology 2003, 38: $560-566$

3. Czauderna P, Haeberle B, Hiyama E, Rangaswami A, Krailo M, Maibach R, Rinaldi E, Feng Y, Aronson D, Malogolowkin M, Yoshimura K, Leuschner I, Lopez-Terrada D, Hishiki T, Perilongo G, von Schweinitz D, Schmid I, Watanabe K, Derosa M, Meyers R; The Children's Hepatic tumors International Collaboration (CHIC): Novel global rare tumor database yields new prognostic factors in hepatoblastoma and becomes a research model. Eur J Cancer 2016, 52: 92-101

4. Perilongo G, Brown J, Shafford E, Brock P, De Camargo B, Keeling JW, Vos A, Philips A, Pritchard J, Plaschkes J: Hepatoblastoma presenting with lung metastases: treatment results of the first cooperative, prospective study of the International Society of Paediatric Oncology on childhood liver tumors. Cancer 2000, 89: $1845-1853$

5. Meyers RL, Rowland JR, Krailo M, Chen Z, Katzenstein HM, Malogolowkin MH: Predictive power of pretreatment prognostic factors in children with hepatoblastoma: a report from the Children's Oncology Group. Pediatr Blood Cancer 2009, 53:1016-1022

6. Cairo S, Armengol C, De Reynies A, Wei Y, Thomas E, Renard CA, et al: Hepatic stem-like phenotype and interplay of Wnt/beta-catenin and Myc signaling in aggressive childhood liver cancer. Cancer Cell 2008, 14:471-484

7. Dan YY, Riehle KJ, Lazaro C, Teoh N, Haque J, Campbell JS, Fausto N: Isolation of multipotent progenitor cells from human fetal liver capable of differentiating into liver and mesenchymal lineages. Proc Natl Acad Sci U S A 2006, 103:9912-9917

8. Koch A, Denkhaus D, Albrecht S, Leuschner I, von Schweinitz D, Pietsch T: Childhood hepatoblastomas frequently carry a mutated degradation targeting box of the beta-catenin gene. Cancer Res 1999, 59:269-273

9. Koch A, Weber N, Waha A, Hartmann W, Denkhaus D, Behrens J, Birchmeier W, von Schweinitz D, Pietsch T: Mutations and elevated transcriptional activity of conductin (AXIN2) in hepatoblastomas. J Pathol 2004, 204:546-554

10. Aretz S, Koch A, Uhlhaas S, Friedl W, Propping P, von Schweinitz D, Pietsch T: Should children at risk for familial adenomatous polyposis be screened for hepatoblastoma and children with 
apparently sporadic hepatoblastoma be screened for APC germline mutations? Pediatr Blood Cancer 2006, 47:811-818

11. Cairo S, Armengol C, Buendia MA: Activation of Wnt and Myc signaling in hepatoblastoma. Front Biosci (Elite Ed) 2012, 4: 480-486

12. Li H, Wolfe A, Septer S, Edwards G, Zhong X, Abdulkarim AB, Ranganathan S, Apte U: Deregulation of Hippo kinase signalling in human hepatic malignancies. Liver Int 2012, 32:38-47

13. Camargo FD, Gokhale S, Johnnidis JB, Fu D, Bell GW, Jaenisch R, Brummelkamp TR: YAP1 increases organ size and expands undifferentiated progenitor cells. Curr Biol 2007, 17:2054-2060

14. Tan X, Behari J, Cieply B, Michalopoulos GK, Monga SP: Conditional deletion of beta-catenin reveals its role in liver growth and regeneration. Gastroenterology 2006, 131:1561-1572

15. Monga SP, Pediaditakis P, Mule K, Stolz DB, Michalopoulos GK: Changes in WNT/beta-catenin pathway during regulated growth in rat liver regeneration. Hepatology 2001, 33:1098-1109

16. Tao J, Calvisi DF, Ranganathan S, Cigliano A, Zhou L, Singh S, Jiang L, Fan B, Terracciano L, Armeanu-Ebinger S, Ribback S, Dombrowski F, Evert M, Chen X, Monga SP: Activation of betacatenin and Yap1 in human hepatoblastoma and induction of hepatocarcinogenesis in mice. Gastroenterology 2014, 147:690-701

17. Bauer M, Eickhoff JC, Gould MN, Mundhenke C, Maass N, Friedl A: Neutrophil gelatinase-associated lipocalin (NGAL) is a predictor of poor prognosis in human primary breast cancer. Breast Cancer Res Treat 2008, 108:389-397

18. Candido S, Maestro R, Polesel J, Catania A, Maira F, Signorelli SS, McCubrey JA, Libra M: Roles of neutrophil gelatinase-associated lipocalin (NGAL) in human cancer. Oncotarget 2014, 5:1576-1594

19. Rodvold JJ, Mahadevan NR, Zanetti M: Lipocalin 2 in cancer: when good immunity goes bad. Cancer Lett 2012, 316:132-138

20. Roli L, Pecoraro V, Trenti T: Can NGAL be employed as prognostic and diagnostic biomarker in human cancers? a systematic review of current evidence. Int J Biol Markers 2017, 32:e53-e61

21. Goetz DH, Holmes MA, Borregaard N, Bluhm ME, Raymond KN, Strong RK: The neutrophil lipocalin NGAL is a bacteriostatic agent that interferes with siderophore-mediated iron acquisition. Mol Cell 2002, 10:1033-1043

22. Wheeler DS, Devarajan P, Ma Q, Harmon K, Monaco M, Cvijanovich N, Wong HR: Serum neutrophil gelatinase-associated lipocalin (NGAL) as a marker of acute kidney injury in critically ill children with septic shock. Crit Care Med 2008, 36:1297-1303

23. Mishra J, Ma Q, Kelly C, Mitsnefes M, Mori K, Barasch J, Devarajan P: Kidney NGAL is a novel early marker of acute injury following transplantation. Pediatr Nephrol 2006, 21:856-863

24. Devarajan P: NGAL in acute kidney injury: from serendipity to utility. Am J Kidney Dis 2008, 52:395-399

25. Yang J, Bielenberg DR, Rodig SJ, Doiron R, Clifton MC, Kung AL, Strong RK, Zurakowski D, Moses MA: Lipocalin 2 promotes breast cancer progression. Proc Natl Acad Sci U S A 2009, 106:3913-3918

26. Fernandez CA, Yan L, Louis G, Yang J, Kutok JL, Moses MA: The matrix metalloproteinase-9/neutrophil gelatinase-associated lipocalin complex plays a role in breast tumor growth and is present in the urine of breast cancer patients. Clin Cancer Res 2005, 11:5390-5395

27. Wang Y, Zeng T: Neutrophil gelatinase-associated lipocalin protein as a biomarker in the diagnosis of breast cancer: a meta-analysis. Biomed Rep 2013, 1:479-483

28. Tong Z, Kunnumakkara AB, Wang H, Matsuo Y, Diagaradjane P, Harikumar KB, Ramachandran V, Sung B, Chakraborty A, Bresalier RS, Logsdon C, Aggarwal BB, Krishnan S, Guha S: Neutrophil gelatinase-associated lipocalin: a novel suppressor of invasion and angiogenesis in pancreatic cancer. Cancer Res 2008, 68:6100-6108

29. Xu B, Jin DY, Lou WH, Wang DS: Lipocalin-2 is associated with a good prognosis and reversing epithelial-to-mesenchymal transition in pancreatic cancer. World J Surg 2013, 37:1892-1900

30. Iannetti A, Pacifico F, Acquaviva R, Lavorgna A, Crescenzi E, Vascotto C, Tell G, Salzano AM, Scaloni A, Vuttariello E,
Chiappetta G, Formisano S, Leonardi A: The neutrophil gelatinaseassociated lipocalin (NGAL), a NF-kappaB-regulated gene, is a survival factor for thyroid neoplastic cells. Proc Natl Acad Sci U S A 2008, 105:14058-14063

31. Mahadevan NR, Rodvold J, Almanza G, Perez AF, Wheeler MC, Zanetti M: ER stress drives Lipocalin 2 upregulation in prostate cancer cells in an NF-kappaB-dependent manner. BMC Cancer 2011, 11:229

32. Kim HJ, Ohk B, Kang WY, Seong SJ, Suk K, Lim MS, Kim SY, Yoon YR: Deficiency of lipocalin-2 promotes proliferation and differentiation of osteoclast precursors via regulation of c-Fms expression and nuclear factor-kappa B activation. J Bone Metab 2016, 23:8-15

33. Tang HC, Chang PC, Chen YC: Iron depletion strategy for targeted cancer therapy: utilizing the dual roles of neutrophil gelatinaseassociated lipocalin protein. J Mol Model 2016, 22:32

34. Gautier L, Cope L, Bolstad BM, Irizarry RA: affy-analysis of Affymetrix GeneChip data at the probe level. Bioinformatics 2004, 20:307-315

35. Sandberg R, Larsson O: Improved precision and accuracy for microarrays using updated probe set definitions. BMC Bioinformatics 2007, 8:48

36. Ritchie ME, Phipson B, Wu D, Hu Y, Law CW, Shi W, Smyth GK: limma powers differential expression analyses for RNA-sequencing and microarray studies. Nucleic Acids Res 2015, 43:e47

37. Lachmann A, Xu H, Krishnan J, Berger SI, Mazloom AR, Ma'ayan A: ChEA: transcription factor regulation inferred from integrating genomewide ChIP-X experiments. Bioinformatics 2010, 26:2438-2444

38. Heinz S, Benner C, Spann N, Bertolino E, Lin YC, Laslo P, Cheng JX, Murre C, Singh H, Glass CK: Simple combinations of lineage-determining transcription factors prime cis-regulatory elements required for macrophage and B cell identities. Mol Cell 2010, $38: 576-589$

39. Committee for the Update of the Guide for the Care and Use of Laboratory Animals: National Research Council: Guide for the Care and Use of Laboratory Animals: Eighth Edition. Washington, DC, National Academies Press, 2011

40. Nejak-Bowen K, Moghe A, Cornuet P, Preziosi M, Nagarajan S, Monga SP: Role and regulation of p65/beta-catenin association during liver injury and regeneration: a "complex" relationship. Gene Expr 2017, 17:219-235

41. Lopez-Terrada D, Alaggio R, de Davila MT, Czauderna P, Hiyama E, Katzenstein H, Leuschner I, Malogolowkin M, Meyers R, Ranganathan S, Tanaka Y, Tomlinson G, Fabre M, Zimmermann A, Finegold MJ; Children's Oncology Group Liver Tumor Committee: Towards an international pediatric liver tumor consensus classification: proceedings of the Los Angeles COG liver tumors symposium. Mod Pathol 2014, 27:472-491

42. Guo H, Jin D, Chen X: Lipocalin 2 is a regulator of macrophage polarization and NF-kappaB/STAT3 pathway activation. Mol Endocrinol 2014, 28:1616-1628

43. Wang Y, Zeng TT: Clinical significance of neutrophil gelatinaseassociated lipocalin (NGAL) in colorectal cancer: a meta-analysis Genet Mol Res 2014, 13:7102-7112

44. Ozemir IA, Aslan S, Eren T, Bayraktar B, Bilgic C, Isbilen B, Yalman H, Yigitbasi R, Alimoglu O: The diagnostic and prognostic significance of serum neutrophil gelatinase-associated lipocalin levels in patients with colorectal cancer. Chirurgia (Bucur) 2016, 111: $414-421$

45. Asimakopoulou A, Weiskirchen S, Weiskirchen R: Lipocalin 2 (LCN2) expression in hepatic malfunction and therapy. Front Physiol 2016, 7:430

46. Zhou F, Luo Q, Wang L, Han L: Diagnostic value of neutrophil gelatinase-associated lipocalin for early diagnosis of cardiac surgeryassociated acute kidney injury: a meta-analysis. Eur J Cardiothorac Surg 2016, 49:746-755

47. Bell D, Ranganathan S, Tao J, Monga SP: Novel advances in understanding of molecular pathogenesis of hepatoblastoma: a Wnt/beta-catenin perspective. Gene Expr 2017, 17:141-154 
48. Kienzl-Wagner K, Moschen AR, Geiger S, Bichler A, Aigner F, Brandacher G, Pratschke J, Tilg H: The role of lipocalin-2 in liver regeneration. Liver Int 2015, 35:1195-1202

49. Xu MJ, Feng D, Wu H, Wang H, Chan Y, Kolls J, Borregaard N, Porse B, Berger T, Mak TW, Cowland JB, Kong X, Gao B: Liver is the major source of elevated serum lipocalin-2 levels after bacterial infection or partial hepatectomy: a critical role for IL-6/STAT3. Hepatology 2015, 61:692-702

50. Kim SL, Lee ST, Min IS, Park YR, Lee JH, Kim DG, Kim SW: Lipocalin 2 negatively regulates cell proliferation and epithelial to mesenchymal transition through changing metabolic gene expression in colorectal cancer. Cancer Sci 2017, 108:2176-2186

51. Feng M, Feng J, Chen W, Wang W, Wu X, Zhang J, Xu F, Lai M: Lipocalin2 suppresses metastasis of colorectal cancer by attenuating NF-kappaB-dependent activation of snail and epithelial mesenchymal transition. Mol Cancer 2016, 15:77

52. Wang YP, Yu GR, Lee MJ, Lee SY, Chu IS, Leem SH, Kim DG: Lipocalin-2 negatively modulates the epithelial-to-mesenchymal transition in hepatocellular carcinoma through the epidermal growth factor (TGF-beta1)/Lcn2/Twist1 pathway. Hepatology 2013, 58:1349-1361 